\title{
The Effects of Solar Activity and Geomagnetic Disturbance on Human Health
}

\author{
Abdullrahman H Maghrabi'* and Marwa A Maghrabi ${ }^{2}$ \\ ${ }^{1}$ National Centre for Applied Physics, King Abdulaziz City for Science and Technology, Saudi Arabia \\ ${ }^{2}$ IMSI University, Saudi Arabia
}

\begin{abstract}
Heliobiology is a new branch of science that deals with the influences on human health caused by solar activity and investigates the possible mechanisms to explain the reported associations. In the last decades, many researchers have considered geomagnetic storms, cosmic rays, and solar flares to be hazardous to human health. They have established that these space weather indicators could play a role in regulating external factors in human health. Heliobiological results have attracted scientists from various branches of science to do more work in this field. In this short paper, the concepts of solar activity, space weather, and heliobiology will be briefly introduced and discussed. The main findings of the effects of solar activity on human health will be summarized.
\end{abstract}

KEYWORDS: Space weather; Solar activity; Heliobiology; Human health

\section{INTRODUCTION}

The sun is the main source of energy for life on Earth. Without its heat and light, life would not exist. The energy generated inside the sun through nuclear fusion reaches us as electromagnetic radiation, which covers all wavelengths from gamma rays to radio waves. The Earth's atmosphere absorbs almost all of the sun's gamma rays and $\mathrm{X}$-rays and part of the ultraviolet radiation. At certain geographical location, solar radiation is affected by astronomical factors, such as season, day of the year, and meteorological conditions (e.g., temperature, humidity, clouds, and aerosols) [1]. In addition to the electromagnetic radiation and in quiet conditions, the sun expels a constant amount of solar plasma and magnetic fields, known as solar winds. At the Earth's orbit, the solar wind has a density of about $3 \mathrm{~cm}-3$, a mean velocity of about $400 \mathrm{~km} / \mathrm{s}$, and a mean magnetic field of about $5 \mathrm{nT}[2,3]$.

The sun is an active star with an 11-year periodic variation known as a solar activity cycle. During this period, the sun undergoes changes characterized by variations in its output of electromagnetic radiation and charged particles due to the different degrees of activity. Each cycle is divided into three phases: the rise phase, with an increasing range of active phenomena; the maximum phase as the phenomena peak; and the decline phase as they gradually decay to the quiet sun levels. Although solar activity accounts for only $1 \%$ of the energy produced by the sun, it exerts a significant influence on the Earth and its surrounding environment [4,5]. During the solar activity period, the number of sunspots increase and galactic cosmic rays decrease. In addition, solar disturbances, large amounts of X-ray radiation, and energetic events (e.g., solar flares, coronal mass ejections, solar energetic particle events, and high-speed solar wind streams) are frequently observed [6]. These events result a considerable amount of radiation; highly energetic particle fluxes and magnetic fluxes are released into the interplanetary medium [2,5]. The Earth' atmosphere, which protects it from dangerous electromagnetic radiation, is surrounded by a magnetic field, known as the magnetosphere, which protects it from charged particles. The Earth and its surroundings are very sensitive to changes in solar activity [7]. When solar events occur, major disturbances affect the Earth's magnetosphere, causing geomagnetic storms and perturbations in the upper atmosphere (mainly the ionosphere) that may be considered potential hazards for terrestrial processes [8]. The sun is the main driver of the state of near-Earth space on all spatial and temporal scales. Spatial scales extend from the

\begin{tabular}{|c|c|}
\hline \multirow[t]{2}{*}{ Quick Re } & $\begin{array}{l}\text { Address for correspondence: Abdullrahman H Maghrabi, National Centre for } \\
\text { Applied Physics, King Abdulaziz City for Science and Technology, Saudi Arabia }\end{array}$ \\
\hline & $\begin{array}{l}\text { Received: July 22, } 2020 \quad \text { Published: August 06, } 2020 \\
\text { How to cite this article: Abdullrahman HM, Marwa AM. The Effects of Solar Activity and } \\
\text { Geomagnetic Disturbance on Human Health. } 2020 \text { - 2(4) OAJBS.ID.000203. DOI: } 10.38125 / \\
\text { OAJBS.000203 }\end{array}$ \\
\hline
\end{tabular}


interplanetary medium, the magnetosphere, and the ionosphere Temporal scales range from 1 to 5 days for propagation of solar wind from the sun to the earth, depending on the velocity of the interplanetary structure imposed on the terrestrial magnetosphere, to a few hours for solar energetic particles events or 8 minutes for electromagnetic radiation [2].

\section{HELIOPHYSICS}

Space weather (or heliophysics) is a branch of space physics that describes the conditions on the sun, solar wind, interplanetary magnetic fields, the magnetosphere, and the ionosphere. With the advancement in technology during the 20th century and due to the disturbances caused by solar activity that affects the performance and reliability of space-born and ground-based technological systems, the interest in space weather has increased and it has become an important branch of science [9]. The effects of space weather phenomena are many and varied. They include electronic and power transmission failures, interruptions in telecommunications and navigational systems, hazards to astronauts, aircraft crews, and passengers, and disruptions of oil and gas pipelines, railway traffic, and human health [7,8,10-13].

Space weather data are collected from ground-based detectors and operational satellites. Data from ground-level stations include cosmic ray data from neutron monitors the number of sunspots, total electron content, and solar flux at $10.7 \mathrm{~cm}$ radio frequencies. Moreover, magnetic storms and the level of geomagnetic activity are recorded by magnetometers through the global network of magnetic field observatories. Various indices, such as Kp, Ap, and Dst, have been introduced to represent geomagnetic activity [5].

On the other hand, several orbital and geostationary satellites conduct space weather observations from space. They include NASA's Advanced Composition Explorer satellite, which has monitored solar wind parameters (velocity, density, and temperature) since 1997. In addition, the Geostationary Operational Environmental Satellite measures the solar X-ray flux from solar flares in two frequency bands and records the charged particles from coronal mass ejections. The Deep Space Climate Observatory satellite is a space weather satellite that launched in February 2015 to give advance warning of coronal mass ejections $[5,8]$.

\section{HELIOBIOLOGY}

Heliobiology [13] (sometimes referred to as cosmobiology, heliomedicine or clinical cosmobiology in the literature) is a new branch of science that deals with the influences on human health caused by solar activity and investigates the possible mechanisms to explain the reported associations.

Over the last 20 years, heliobiology has become a subject of interest that has attracted scientists from various disciplines. Numerous studies have been carried out, and the evidences suggest that space weather activity has a broad range of adverse effects on human health, such as mental illness, cardiovascular mortality, and neurological system diseases [14-16]. On the other hand, other studies have reported no such of relationships exist [15]. Although contradictions have been found between these studies, this field of research has become increasingly important in the present context of a consistent weakening of the Earth's magnetic field.

One challenging problem (more of a controversial issue) in heliobiology is the search for mechanisms by which different events and processes on the sun can have either direct or indirect effects on human health and physiology. Although several possible mechanisms have been proposed to explain this connection, there is widespread skepticism about the reality of such relationships.

One of the proposed mechanisms is the effect of geomagnetic storms on the Earth's electric and magnetic fields. It is wellestablished that all biological systems on Earth are exposed to external and internal fluctuating magnetic and electric fields of a wide range of frequencies $(0.1-10 \mathrm{~Hz})$ [15,17-18]. The oscillations over these frequencies are close to the frequency of many internal organs. These oscillations, which are active by the magnetosphere and ionosphere due to geomagnetic storms resulting from solar activity events, may in a resonant way act on the function of living organisms at a cellular level to a greater or lesser degree. The fundamental difficulty when studying the effect of natural electric and magnetic field variations on human health is the fact that the field strengths involved are very small. When the geomagnetic environment is disturbed, it seems plausible that this could have either a direct or an indirect effect on human physiology [17-19].

Another possible mechanism to explain the association of geomagnetic disturbances and human behaviour (depression) involves the alteration of melatonin levels in the body due to geomagnetic disturbances [18, 20-21].

The effects of space weather activities on human health have been qualitatively and quantitatively examined using two quantifiable measures: the direct and indirect indicators. The indirect indicators are long-term medical data, such as the temporal distribution of emergency calls and hospital admissions [15].

The relationship between indirect indicators and space weather indicators is usually investigated using two statistical approaches:

a) Correlation analyses and their associated statistical tests between the time series of the space weather variables and medical data $[15,22]$.

b) Cyclic variations to investigate the common periodicities between medical data and space weather data over wide ranges of frequencies using appropriate analytical methods (e.g., Fourier transform) [23].

c) The direct indicators, on the other hand, include all the physiological parameters that can be verified and are acquired either in vivo, directly from the subject (e.g., heart rate variability, blood pressure measurements, microcirculation parameters, and reaction time), or in vitro by laboratory diagnostics or tissue investigations.

\section{RESULTS INVESTIGATIONS FROM HELIOBIOLOGICAL}

Over the last 20 years, several research papers have presented the results of investigating the relationships between space weather parameters and human health. Some of these results are summarized below $[13,15,24]$ :

a) High values of geomagnetic activity have a negative effect on human cardiovascular health that includes significant variations in heart rate variability $[13,25]$.

b) The number of incidents of alterations in blood flow is increased (increased systolic and diastolic blood pressure and epileptic seizures) during the solar activity periods [24,26].

c) Incidents of coronary disease and myocardial infarction increase during spans of high solar activity, as compared to years with low solar activity [13-15-16]. 
d) Sharp or sudden variations in geomagnetic and solar activity can act as stressors, which alter regulatory processes such as breathing, reproductive, and increase total deaths total deaths [13].

e) Several studies support the idea that geomagnetic disturbances decrease the melatonin levels in the human body [2021].

f) Positive correlations exist between neurological system diseases (e.g., depression and mental illness) and geomagnetic activity [12-13,27-28].

g) The standard metabolism and behaviour patterns of humans and other species are affected by solar activity [13,29-30].

h) Solar disturbances are associated with significant increases in hospital admissions for suicide attempts, homicides, and traffic accidents [12,31].

i) Investigations of the blood of tested patients have shown that the viscosity of blood during solar activity periods increases sharply, so the risk of developing morbid cardiovascular system disease is increased [32].

j) A relationship between solar activity and some congenital anomalies such as Down syndrome has been established [33-34].

k) The fluctuations in solar activity are associated with oscillations in concentrations of vitamin D [35].

l) Solar activity is related to many parameters of new-born development and homeostasis, such as number of births, number of premature births, new-born weight and length, and syndromes associated with chromosome aberrations and hormone production [36-37].

m) Solar activity may contribute to the development of and be a trigger of the exacerbation of nervous and mental disorders, such schizophrenia, Alzheimer's disease, and multiple sclerosis [38].

\section{CONCLUSION}

The results from heliobiological investigations carried out in the last 20 years have reported evidence that suggests solar activity has direct or indirect influences on human health. Although there are speculations about the reality of such relationships, the results have attracted the scientific community to heliobiology and encouraged them to conduct more research in this field and search for mechanisms that can explain such relationships. For more conclusions to be made in the field of heliobiology, more investigations and medical data from different places around the world are needed. In this regard, a national-scale project led by the first author of this paper has been proposed and submitted to the Ministry of Health and other research organizations In Saudi Arabia. The main aim of this project is to investigate the effects of solar activity parameters on physiological and mental disorders and other parameters related to human health status in Saudi Arabia.

\section{REFERENCES}

1. Maghrabi A (2017) The influence of dust storms on solar radiation data, aerosol properties and meteorological variables in Central Arabian Peninsula. Int J Environ Sci Technol 14: 1643-1650.

2. Sakurai K (1987) Cosmic rays and energetic particles in the heliosphere. In: Akasofu SI, Kamide Y (Eds.), The solar wind and the earth. Terra Scientific Publishing Company.
3. Zenchenko T (2011) Solar wind density variations and the development of heliobiological effects during magnetic storms. Atmos Oceanic Phys 47(7):795-804.

4. Usoskin I (2008) A history of solar activity over millennia. Living Rev Sol Phys 5: 3.

5. Dorman L (2004) Cosmic rays in the earth's atmosphere and underground, Kluwer Academic Publishers. The Netherlands.

6. Cane H (1999) Cosmic ray modulation and the solar magnetic field. Geophys Res Lett 26: 565-568.

7. Maghrabi A, Kudela K (2019) Relationship between time series Cosmic Ray data and Aerosol optical Properties: 1999-2015. J Solar Terrestrial Physics 190: 36-44.

8. Dorman L (2008) Space storms as natural hazards. Adv Geosci 14: 271275.

9. Pandit D (2018) Solar activities and its impact on space weather Proceedings of the International Astronomical Union 13(S340): 149150.

10. Breus T (2008) Some aspects of the biological effects of space weather. J Atmos Sol Terr Phys 70(2-4): 436-441.

11. Mendoza B, Pena S (2009) Solar activity and human health at middle and low geomagnetic latitudes in Central America. Adv Space Res 46(4): 449-459.

12. Sidyakin V (1983) Sensitivity of the nervous system to changes in solar activity (literature review)," Zh Nevrol Psikhiat 83(1): 134-137.

13. Stoupel E (2019) 50 Years in research on space weather effects on human health (Clinical Cosmobiology). EC Cardiology 11: 470-478.

14. Breus T, Binhi V, Petrukovich A (2016) Magnetic factor of the solar terrestrial relations and its impact on the human body: Physical problems and prospects for research. Phys Usp 59: 502-510.

15. Palmer S, Rycroft M, Cermack M (2006) Solar and geomagnetic activity, extremely low frequency magnetic and electric fields and human health at the earth's surface. Surv Geophys 27: 557-595.

16. Vencloviene J, Babarskiene R, Slapikas, R (2013) The association between solar particle events, geomagnetic storms, and hospital admissions for myocardial infarction. Nat Hazards 65: 1-12.

17. Durand-Manterola H, Mendoza B, Diaz-Sandoval R (2001) Electric currents induced inside biological cells by geomagnetic and atmospheric phenomena. Adv Space Res 28(4): 679-684.

18. Krylov V (2017) Biological Effects Related to Geomagnetic Activity and Possible Mechanisms. Bioelectromagnetics 38(7): 497-510.

19. Cherry N (2002) Schumann resonances, a plausible biophysical mechanism for the human health effects of solar/geomagnetic activity. Nat Hazards 26(3): 279-331.

20. Burch J, Reif J, Yost M (2008) Geomagnetic activity and human melatonin metabolite excretion. Neurosci Lett 438(1): 76-79.

21. Weydahl A (2001) Geomagnetic activity influences the melatonin secretion at 70 degrees N. Biomed Pharmocother 55(Suppl 1): 57-62.

22. Lomb N (1976) Least-squares frequency analysis of unequally spaced data. Astrophys Space Sci 39(2): 447-462.

23. Scargle J (1982) Studies in astronomical time series analysis. II. Statistical aspects of spectral analysis of unevenly spaced data. Astrophys J 263: 835-853.

24. Babayev S, Allahverdiyevab A (2007) Effects of geomagnetic activity variations on the physiological and psychological state of functionally healthy humans: Some results of Azerbaijani studies. Advances in Space Research 40(12): 1941-1951.

25. Chernouss SA (2003) The possibility of assessment of heliogeophysical impact on human health by heart rate variability. J Karazin KhNU Series Med 5: 90-91. 
26. Dimitrova S, Stoilova I, Cholakov I (2004) Influence of local geomagnetic storms on arterial blood pressure. Bioelectromagnetics 25:408-414.

27. Meshcheriakova S, Breus T, Sosnovskii A (1998) Magnetic storms as a stress factor. Biofizika 43(4): 632-639.

28. Mulligan B, Persinger M (2012) Experimental simulation of the effects of sudden increases in geomagnetic activity upon quantitative measures of human brain activity: validation of correlational studies. Neurosci Lett 516(1): 54-56.

29. Breus T, Boiko E, Zenchenko T (2015) Magnetic storms and variations in hormone levels among residents of north polar area Svalbard. Life Sci Space Res 4: 17-21.

30. Stoupel E (1995) Relationship between immunoglobulin levels and extremes of solar activity. International Journal of Biometeorology 38(2): 89-91.

31. Kancírová M, Kudela K (2014) The relationship between suicide incidents in Slovakia and the Czech Republic and heliophysical parameters: empirical results. J Astrobiol Outreach 2(2): 1-5.

32. Stoupel E, Joshua H, Lahav J (1996) Human blood coagulation and geomagnetic activity. Eur J Int Med 7:217-220
33. Stoupel E (2005) Chromosome aberration and environmental physical activity: Down syndrome and solar and cosmic ray activity. Israel 1990 2000. International Journal of Biometeorology 50(1): 1-5.

34. Stoupel E (2009) Congenital heart disease: Correlation with fluctuations in cosmophysical activity, 1995-2005. International Journal of Cardiology 135: 207-210.

35. Jackman C, McPeters R (2004) The Effect of solar proton events on ozone and other constituents. In: Solar variability and its effects on climate 141: 305-319.

36. Galpern G (1995) Solar activity and the incidence of foetal chromosome abnormalities detected at prenatal diagnosis. Int J of Biometeorology 39(2): 59-63.

37. Stoupel E (2006) Monthly new-borns number and environmental physical activity. Medicina 42.2: 238-241.

38. Lõhmus M (2018) Possible biological mechanisms linking mental health and heat-a contemplative review. Int J of Envir Research and Public Health 15(7): 1515. 